\title{
Détachements et tectonique extensive dans le massif hercynien des Rehamna (Maroc)
}

\author{
ABDEL M. AGHZER et RICARDO ARENAS
}

\begin{abstract}
Departamento de Petrología y Geoquímica, Facultad de Ciencias Geológicas, Universidad Complutense, 28040 Madrid, Espagne
\end{abstract}

\begin{abstract}
Résumé - A la suite de sa déformation en régime compressif sous des conditions métamorphiques barroviennes culminant dans le faciès amphibolite supérieur, le massif des Rehamna est affecté par une tectonique extensive. Celle-ci s'est manifestée dans trois zones de détachement (Kef El Mouneb, El Broumi et Ouled Zedness occidental) qui ont assuré le retour à l'équilibre de la croûte épaissie. L'amincissement crustal provoqué par la tectọnique extensive est enregistré par les isogrades qui ont subi une forte condensation. La cinématique des mouvements extensifs est variable avec la profondeur; le déplacement tectonique dirigé du sudouest vers le nordest dans les parties profondes de la chaîne, s'opère par contre du nordouest vers le sudest dans les niveaux supérieurs de l'édifice orogénique.
\end{abstract}

\begin{abstract}
In the Moroccan Hercynian belt, the Rehamna massif has been affected by an important extensional tectonism, developed inmediately after a compressive regime with associated Barrovian metamorphism up to the lower-T part of the amphibolite facies. Three main detachments were developed during the extensional regime (Kef El Mouneb, El Broumi and western Ouled Zednes), which caused an important thinning and can be considered to be the origin of the extreme condensation outlined by the metamorphic zones. The extensional movements were oriented from southwest to northeast in the lower parts of the massif, whereas in the upper parts a main sense of movement from northwest to southeast can be detected.
\end{abstract}

\section{INTRODUCTION}

Le massif hercynien des Rehamna constitue une boutonnière de la Méséta marocaine (Fig. 1). Sa partie méridionale est classiquement subdivisée en trois domaines (Michard, 1982): les Rehamna centraux et orientaux, affectés par un métamorphisme barrovien, et les Rehamna occidentaux, non métamorphiques. Les Rehamna centraux sont séparés des Rehamna occidentaux par la faille dite médiane (Fig. 2), initialement normale (Aghzer, 1994) et réactivée postérieurement en décrochement (Piqué, 1972). Les deux domaines métamorphiques des Rehamna sont séparés par la zone de cisaillement de Ouled Zedness (Fig. 2), dont la nature n'est pas définitivement résolue. Elle est interprétée soit comme un décrochevauchement (Hoeppfner et al., 1975; Piqué et al., 1982; Lagarde et Michard, 1986), soit comme un chevauchement à cinématique oblique (Diot, 1989) ou parallèle à l'axe de la chaîne (Corsini et al., 1988).

La nature du contact entre les deux formations de la couverture paléozoïque des Rehamna centraux (Fig. 3a) est également problématique: selon Piqué (1972), Hoeppfner et al. (1975), Destombes et al. (1982) et Piqué et Michard (1989), les métapélites cambriennes de la formation des Skhour sont localement recoupées en discordance angulaire faible par les matériaux dévoniens sus-jacents de la formation de Kef El Mouneb. Pour Corsini (1988) et Diot (1989), la formation de Kef El Mouneb correspond par contre à une butte témoin des chevauchements orientaux. Dans le secteur oriental, le contact superposant les matériaux dévoniens à ceux du Cambro-Ordovicien (Fig. 3a) est interprété par Jenny (1974) et Hoeppfner (1974) comme un chevauchement. Cornée et al. (1982) remettent en question la nature tectonique de ce contact et suggèrent une continuité stratigraphique entre le Dévonien et le Cambro-Ordovicien.

Les contacts tectoniques précités ont été reconsidérés dans ce travail sur la base d'une étude structurale qui a été réalisée dans la majeure partie des Rehamna centraux et dans la partie occidentale des Rehamna orientaux (Fig. 3a), et qui a permis de mettre en évidence une importante tectonique extensive manifestée sous des conditions métamorphiques rétrogrades.

\section{CADRE GEOLOGIQUE}

Le secteur étudié (Figs 2 et $3 a$ ) du domaine central comprend la nappe cambro-ordovicienne des Skhour, 


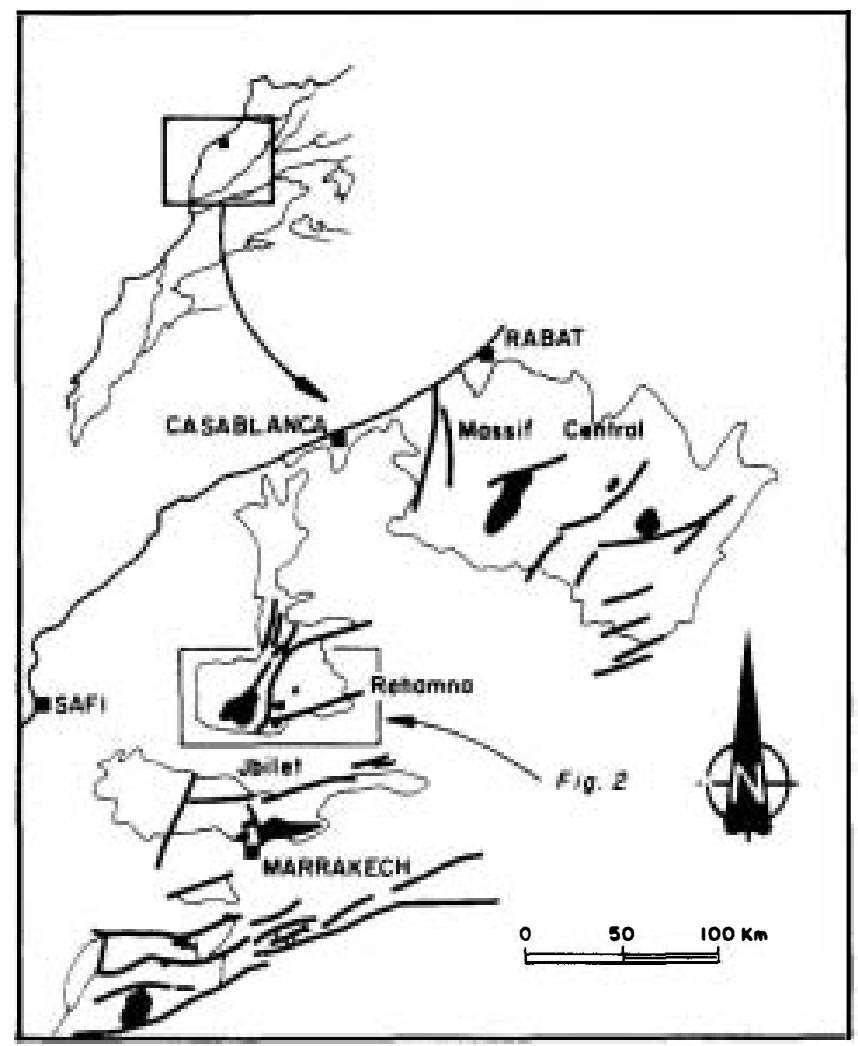

Figure 1. Localisation du massif des Rehamna dans un schéma géologique d’ensemble de la Méséta marocaine.

limitée à sa base par la zone de cisaillement de Kef El Mouneb (ZCKM) qui correspond au toit fortement cisaillé de l'auto- ou para-autochtone (Fig. 3a). Celui-ci comprend des faciès de type 'Vieux Grès Rouges' datés du Dévonien (Corsini, 1988). Le secteur central montre une séquence zonéographique barrovienne (Fig. 4a) allant de la zone de la chlorite (formation des Skhour) ou de la biotite (formation de Kef El Mouneb) jusqu'à la zone de la staurotide (formation des Skhour) ou du disthène (formation de Kef El Mouneb). La zone de cisaillement de Ouled Zedness (ZCOZ) affecte des roches d'âge présumé Dévonien à Tournaisien (Jenny, 1974), affectées par un métamorphisme barrovien à disthène.

Le secteur oriental se situe au sommet de l'édifice structural. Il comprend trois formations lithostrati-graphiques qui sont de bas en haut (Fig. 3a): la formation de Jorf El Ahmar, d'âge CambroOrdovicien (Jenny, 1974), la formation d'El BroumiOuled Hassine (Aghzer, 1994), attribuée au Dévonien (Jenny, 1974), et la formation de Lalla Tittaf (Jenny, 1974), d'âge présumé Tournaisien à Viséen (Aghzer, 1994). Les deux premières formations sont séparées par la zone de cisaillement d'El Broumi (ZCEB; Fig. 3a). L'ensemble de ces formations orientales est af-fecté par un métamorphisme barrovien à staurotide.

\section{CARACTERISTIQUES STRUCTURALES GENERALES}

Le secteur étudié a enregistré trois phases de déformation $D_{1}, D_{2}$ et $D_{3}$. Dans les formations des Skhour et de Jorf El Ahmar, la première phase de déformation a engendré des plis d'extension kilométrique, qui définissent des structures majeures anticlinales de direction NE-SO, déversées vers le nordouest (Fig. 3a; Piqué, 1972; Jenny, 1974; Piqué et al., 1982). Ces plis développent une schistosité de plan axial $S_{1}$ à caractère régional, qui porte une linéation d'étirement subhorizontale, orientée parallèlement aux axes des plis. Dans les autres secteurs du massif, les structures $D_{1}$ sont généralement masquées par les recristallisations associées à la deuxième phase tectonique; les seules structures rencontrées correspondent aux plans $S_{1}$ microplissés lors de la deuxième phase tectonique (Fig. 5b, c).

La deuxième phase tectonique se caractérise par deux régimes tectoniques: un régime compressif, précoce, suivi d'un autre extensif (Aghzer, 1994). Le régime compressif est à l'origine du chevauchement de la formation dévonienne auto- ou para-autochtone de Kef El Mouneb par la formation cambroordovicienne des Skhour, ainsi que du chevauchement des Rehamna centraux par les Rehamna orientaux (Fig. 3). Les structures $D_{2}$ compressives caractérisent régionalement les formations $\mathrm{d}^{\prime} \mathrm{El}$ Broumi-Ouled Hassine et de Lalla Tittaf, alors qu'elles sont restreintes aux bandes étroites de déformation syn-schisteuse, localisées au voisinage immédiat de la ZCKM (cas de la formation des Skhour) et de la limite orientale de la ZCOZ (cas de la formation de Jorf El Ahmar). Il s'agit de plis $\mathrm{P}_{2}$ (Fig. $5 b, c)$ sub-coaxiaux aux plis $P_{1}$, de taille millimétrique à métrique, de type isoclinal à serré. Ces plis sont orientés N-S à NE-SW, montrent un déversement net vers l'ouest à nordouest et présentent un faible plongement axial vers le nord à nordest. Ils développent une schistosité $S_{2}$ de plan axial qui évolue à proximité (cas de la ZCKM) et au niveau (cas de la limite tectonique orientale de la $\mathrm{ZCOZ}$ ) des zones de cisaillement vers une foliation mylonitique. Les critères du sens de cisaillement (Figs $5 d$, e et $6 a$, b) fournis par les métasédiments situés au niveau (cas de la limite orientale de la $\mathrm{ZCOZ}$ ) ou au voisinage (cas de la ZCKM) des zones de cisaillement, indiquent que les mouvements chevauchants sont dirigés de l'est à sudest vers l'ouest à nordouest. Le retour à l'équilibre de la croûte épaissie par ces chevauchements est assuré par la tectonique extensive qui s'est manifestée dans la ZCKM, la ZCEB et la limite occidentale de la ZCOZ (Fig. 3). Ces zones de cisaillement correspondent à des niveaux de détachement crustaux comparables à ceux associés 


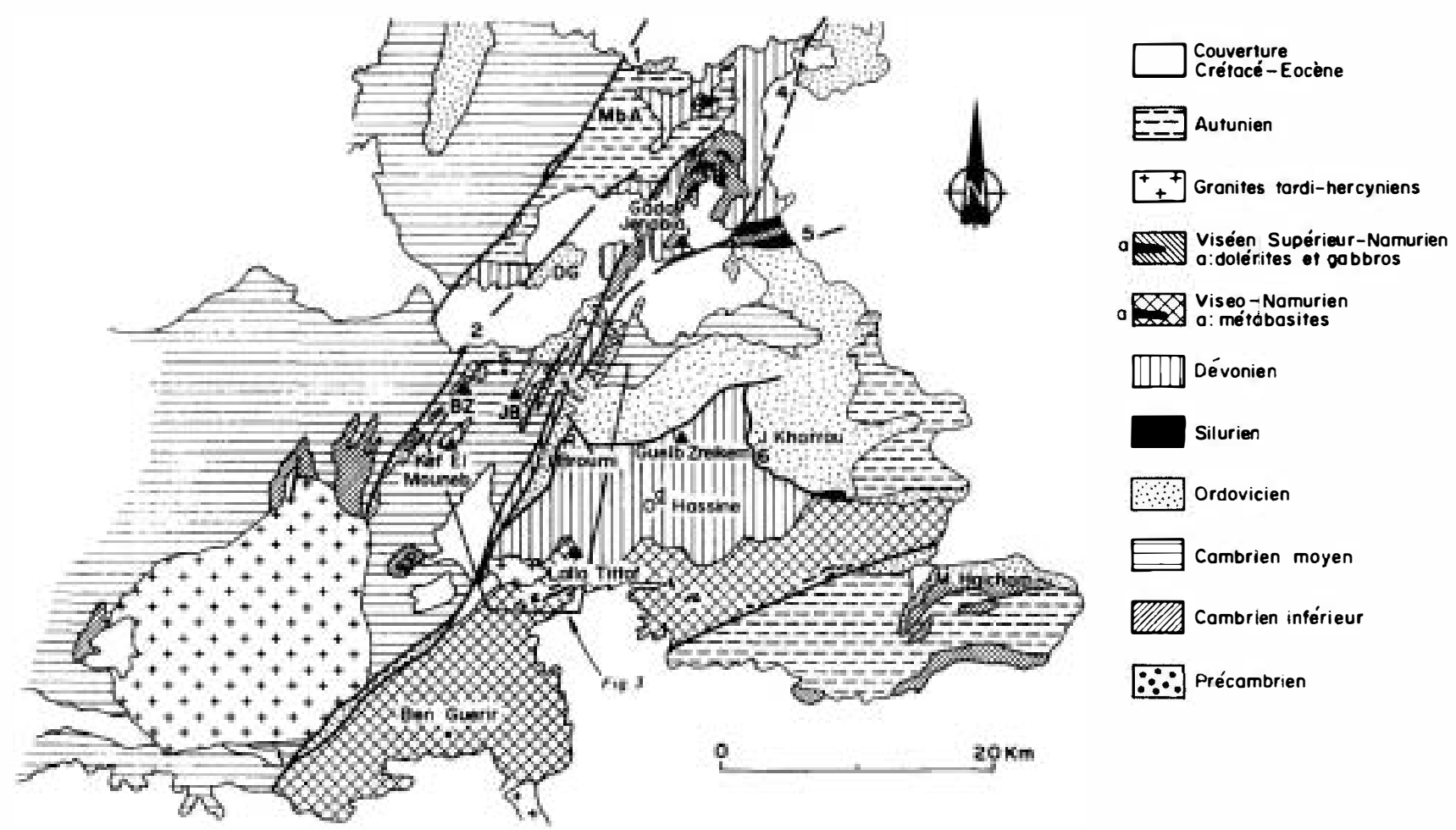

Figure 2. Carte de répartition des formations paléozoïques dans le massif des Rehamna et situation du secteur étudié. Failles: 1-Haut Tarfa; 2-médiane; 3-Ouled Zedness; 4-Kibane; 5-Aïn Mellah; 6-Jbel Kharrou; S-Skhour Rehamna; MbA-Mechraa ben Abbou; DG-Draa Guessa; BZ-Baten Zita; JB-Jorf el Beida. (d'après Michard, 1982 et Corsini, 1988).

aux complexes métamorphiques profonds (Davis, 1980; Coney, 1984; Hodges et al., 1987; Malavieille, 1987; Sandiford, 1989; Sonder et al., 1987; Escuder Viruete et al., 1994). Elles ont donné lieu à des structures planaires pénétratives, globalement parallèles en orientation aux fabriques planaires compressives syn- $D_{2}$. Les critères du sens de cisaillement selon leurs linéations d'étirement indiquent une variation cinématique des mouvements extensifs qui se sont opérés selon deux directions: du sudouest vers le nordest (Figs $5 \mathrm{f}$ et $6 \mathrm{c}$, d) et du nordouest vers le sudest (Fig 6e, $\mathrm{f}$ ).

L'ensemble des structures antérieures est ensuite repris par la troisième phase tectonique qui a conféré au massif sa géométrie d'ensemble. Dans le secteur central, cette phase tectonique a donné naissance à un anticlinal $\mathrm{P}_{3}$ coaxial aux plis $\mathrm{P}_{1}$ de la formation des Skhour (Corsini, 1988; Aghzer, 1994), droit à déversé vers le nordouest, à plongement axial faible vers le nordest (Fig. 3). Dans le secteur oriental, le plissement $P_{3}$ a engendré un synclinal de direction $\mathrm{N} 140^{\circ}$ (Fig. 3), à fort plongement axial vers le sudest et à déversement net vers le sudouest. Font également partie des structures $\mathrm{D}_{3}$ des décrochements généralement dextres, qui s'organisent en quatre familles: deux familles conjuguées $\mathrm{N}$ 140$170^{\circ}$ dextre et $\mathrm{N} 80-90^{\circ}$ senestre, une troisième famille orientée NE-SW et enfin une quatrième orientée N-S (Fig. 3a).

\section{METAMORPHISME ASSOCIE A LA DEFORMATION HERCYNIENNE}

La relation entre le métamorphisme et la déformation est illustrée dans la Figure 4 . La première phase et les stades compressifs de la deuxième phase tectonique sont associées à un métamorphisme barrovien dont le paroxysme thermique régional, atteint dans la zone de la staurotide et/ou du disthène, est conditionné par les chevauchements syn- $\mathrm{D}_{2}$ (Aghzer, 1994).

La tectonique extensive tardi- $\mathrm{D}_{2}$ est par contre associée à une évolution métamorphique rétrograde (Aghzer, 1994), de type décompression presque isotherme, qui s'est initiée dans le champ de stabilité du disthène, où elle a donné lieu à des assemblages similaires à ceux de l'événement métamorphique antérieur, et s'est achevée dans celui de l'andalousite. Le caractère rétrograde de cette évolution métamorphique est indiqué par la forte réduction de la taille des grains des roches situées dans les zones de détachement.

Le reste de l'évolution métamorphique rétrograde est associé au plissement de troisieme phase tectonique. Celui-ci s'est manifesté à basse pression depuis les conditions du faciès amphibolite supérieur jusqu'à celles du faciès schistes verts supérieur. $\mathrm{Au}$ cours de cette phase ectonique, la croute, encore peu épaisse, subit une exhumation modérée sous le contrôle de l'érosion (Aghzer, 1994). 


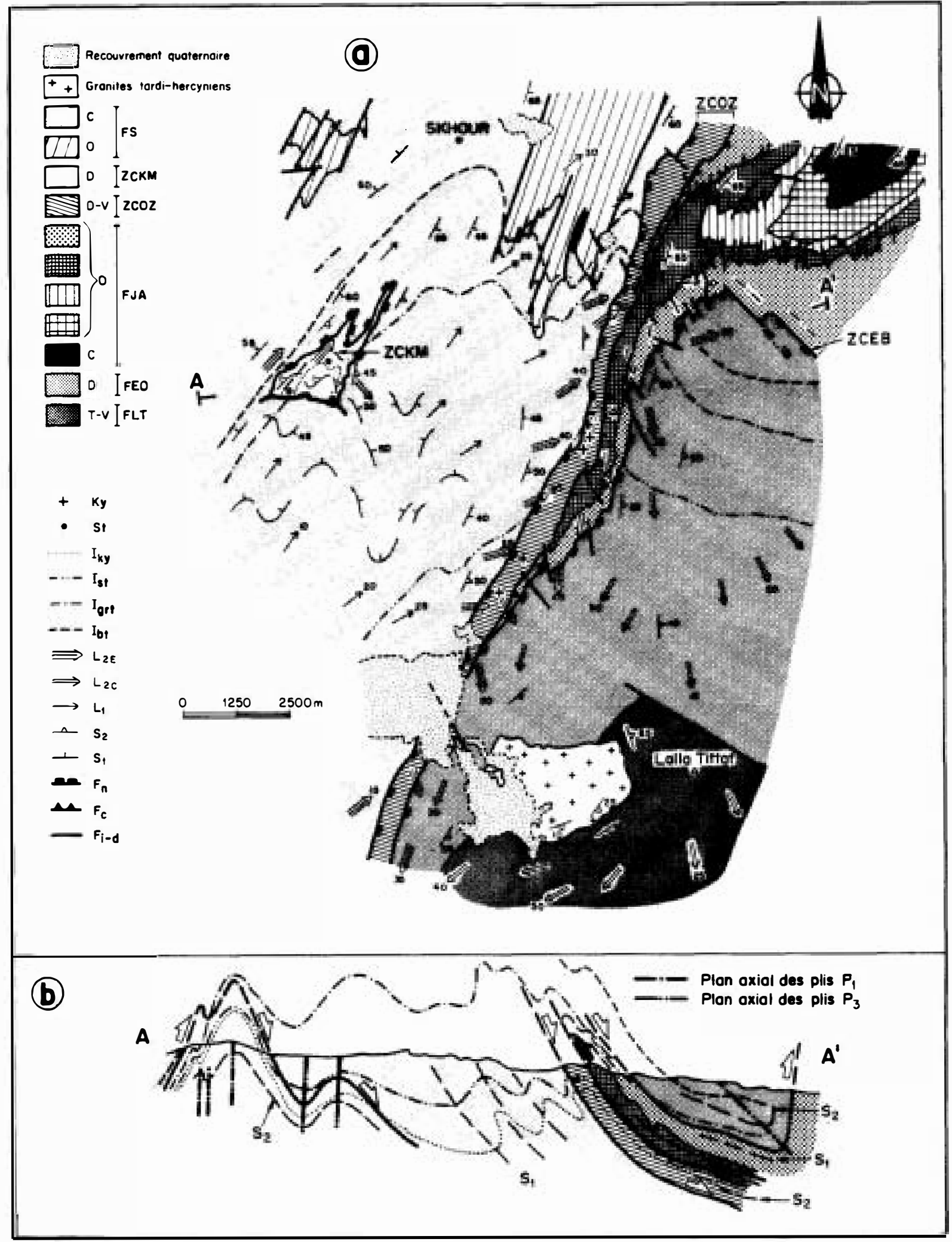

Figure 3. (a) Carte géologique simplifiée du secteur étudié: C-Cambrien; O-Ordovicien; D-Dévonien; D-V-Dévonien à Viséen; T-V-Toumaisien à Viséen; FS-formation des Skhour; ZCKM-zone de cisaillement de Kef El Muneb; ZCOZ-zone de cisaillement de Ouled Zedness; FJA-formation de Jorf El Ahmar; FEO-formation d'El Broumi-Ouled Hassine; FLT-formation de Lalla Tittaf; ky-localités du disthène; St-localités de la staurotide; Iky-isograde du disthène; Ist-isograde de la staurotide; Igrt-isograde du grenat; Ibt-isograde de la biotite; L2E-linéation d'étirement associée à la tectonique extensive tardi- $D_{2}$; $L_{2 C}-$ linéation d'étirement associée aux stades compressifs de deuxième phase tectonique; L1-linéation d'étirement associée à la première phase de déformation; $S_{2}$-plan de schistosité associé à la déformation $D_{2}$; $S_{1}$-plan de schistosité associé à la

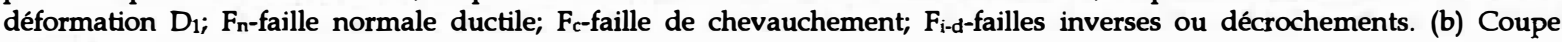
géologique. 


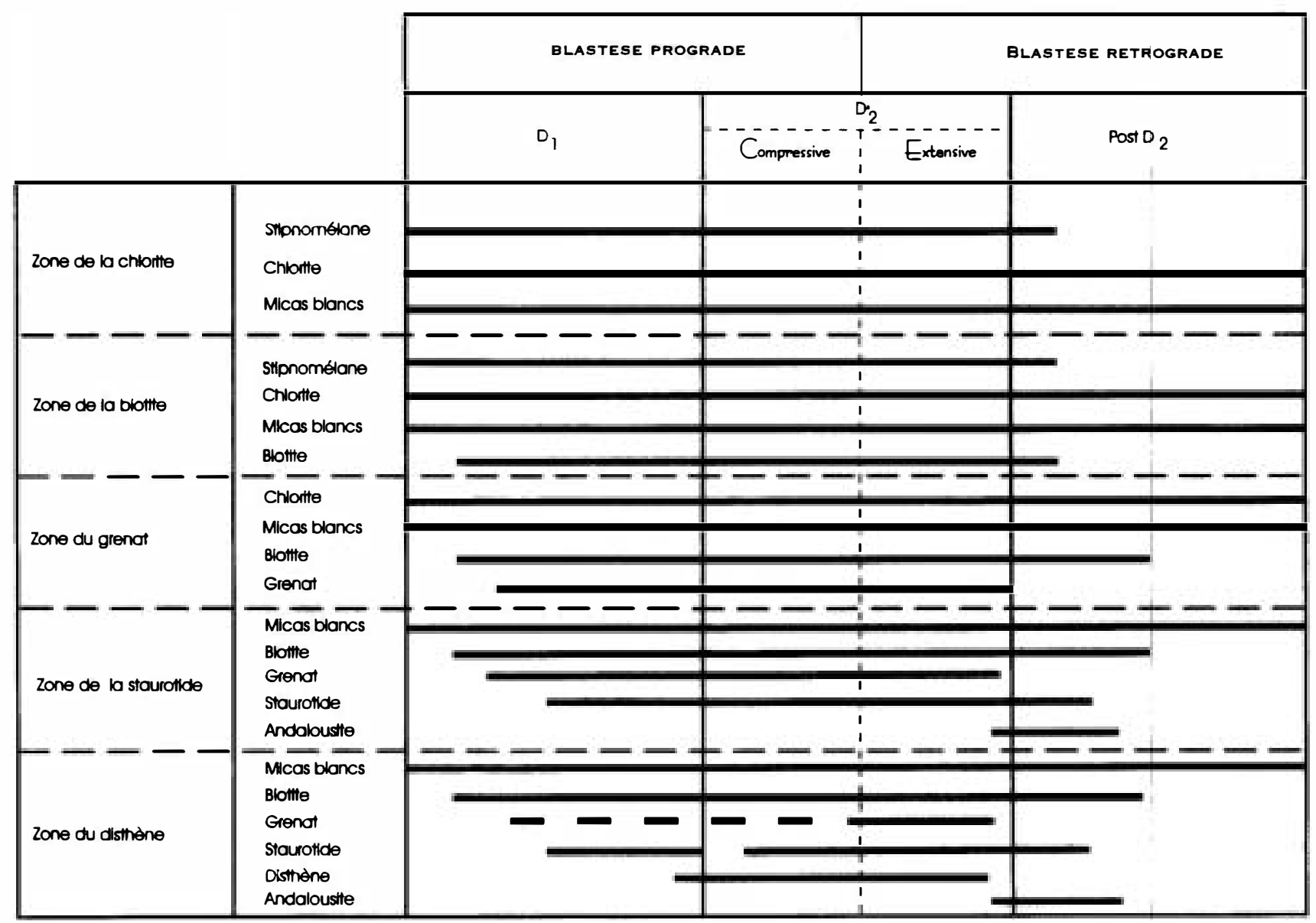

Figure 4. Relation métamorphisme-déformation dans les lithologies pélitiques à semipélitiques.

\section{ZONE DE CISAILLEMENT DE KEF EL MOUNEB (ZCKM)}

La cartographie des plans de schistosité a permis de mettre en evidence, aux environs de Kef El Mouneb, un anticlinal $P_{3}$; celui-ci est ouvert et droit dans les affleurements Sud à fortement aplati et déversé vers le nordouest dans les affleurements nord (Fig. 3a). Selon la coupe géologique de la Figure $3 b$, la formation dévonienne de Kef El Mouneb se situe au dessous de la formation cambro-ordovicienne des Skhour et correspond donc à une fenêtre tectonique. Ce schéma allochtone est appuyé davantage par la présence de paragenèses à staurotide et disthène dans la ZCKM et leur absence dans les métapélites environnantes de la nappe des Skhour (Fig. 3a). L'occurence de ces assemblages dans les matériaux dévoniens suggèrent un épaississement crustal considérable, acquis au cours de leur chevauchement par la formation des Skhour.

En ce qui concerne la disposition localement discordante des matériaux méridionaux de la formation de Kef El Mouneb sur les métapélites cambriennes de la formation des Skhour (Piqué, 1972; Hoeppfner et al., 1975; Destombes et al., 1982; Piqué et Michard, 1989), elle est attribuée à une faille inverse tardive qui recoupe les isogrades plissés par la déformation $D_{3}$ (Fig. 3a). Cette faille n'affecte cependant pas un filon de dacitoïde qui intrude aussi bien les matériaux cambriens que ceux dévoniens. Son jeu est donc antérieur aux dacitoïdes, d'âge anté-autunien (Hoeppfner et al., 1982), et postérieur au plissement $P_{3}$.

Les matériaux dévoniens sont affectés par une déformation extrêmement pénétrative, matérialisant l'existence d'une zone de cisaillement (ZCKM) correspondant au toit de la formation auto- ou paraautochtone de Kef El Mouneb. Les structures associées à la ZCKM sont principalement engendrées par la tectonique extensive tardi- $\mathrm{D}_{2}$ qui a presque complètement oblitéré les structures compressives antérieures.

\section{Structures associées à la tectonique compressive}

Elles correspondent aux plis $P_{2}$ préservés localement dans des domaines relativement compétents. Il $s^{\prime}$ agit de plis isoclinaux de taille centimétrique à milimétrique, de direction axiale N-S à NE-SW. Ils présentent un faible plongement axial vers le nord à nordest et sont déversés vers l'ouest à nordouest. Ces plis ont été également rencontrés dans une bande étroite de déformation syn-schisteuse limitant la base 

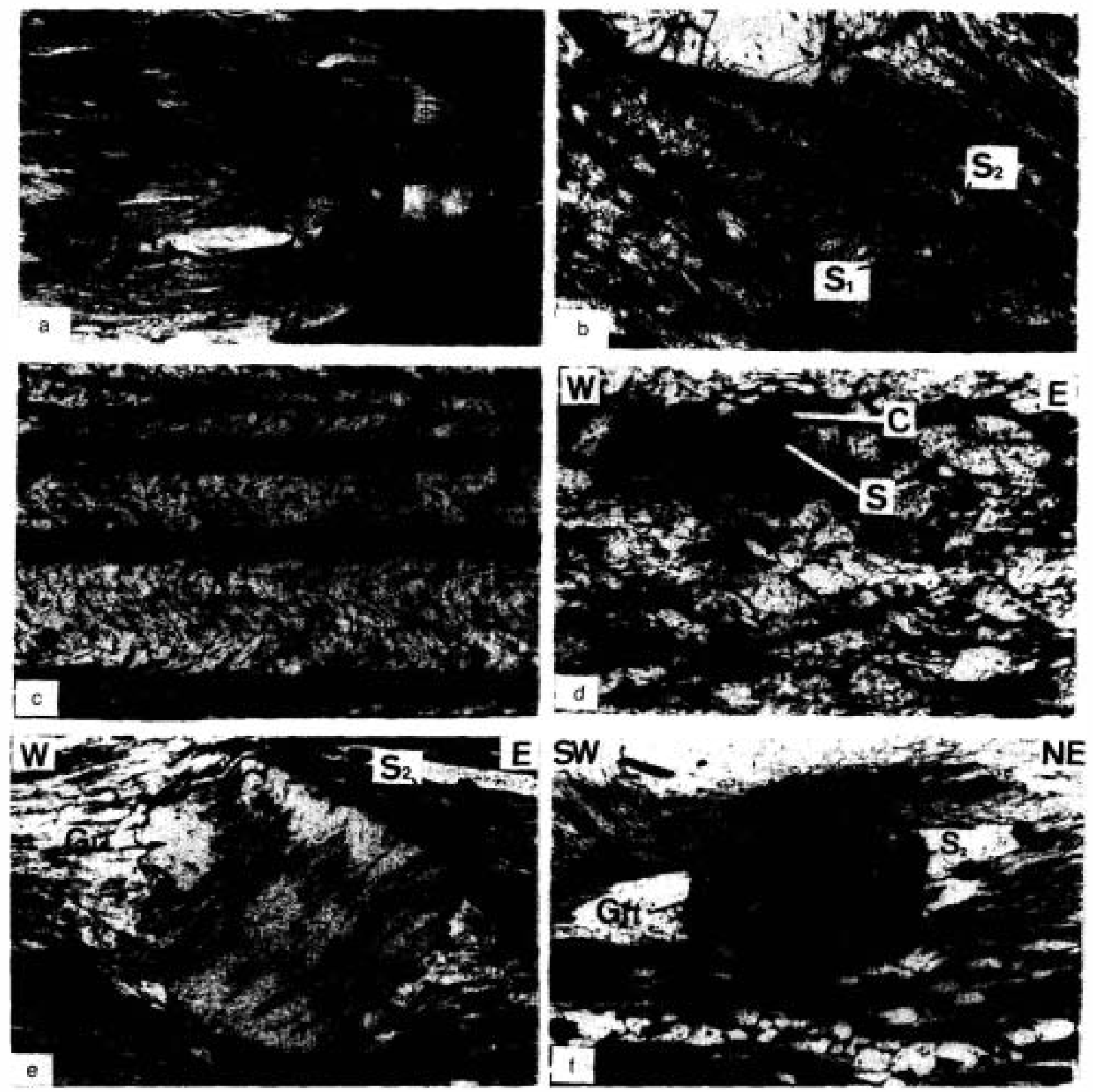

Figure 5. (a) Linéation d'étirement indiquée par le grand axe des galets des métaconglomérats de la ZCKM. (b) Schistosité de crénulation de deuxième phase de déformation, développée dans les métapélites de la formation d’El Broumi-Ouled Hassine. (c) Schistosité de crénulation de deuxième phase de déformation, développée dans les métapélites de la formation des Skhour. (d) Structures S-C développées dans les métagrawauckes localisées près du contact basal de la nappe des Skhour, produits des stades compressifs de la deuxième phase tectonique. Le sens de cisaillement qu'elles indiquent suggère un déplacement de la formation des Skhour de l'est vers l'ouest. (e) Grenat syn-S compressive, développé dans les métapélites basales de la nappe des Skhour. Il englobe des figures de rotation syn-cinématique suggérant une translation de la formation des Skhour de l'est vers l'ouest. (f) Grenat syn-S2 extensive, développé dans les métapélites de la ZCKM. Sa croissance en contexte rotationnel indique un déplacement du bloc supérieur (formation des Skhour) du sudouest vers le nordest. Grt-grenat. La barre noire mesure $150 \mathrm{~mm}$.

de la formation des Skhour. Ils développent une schistosité de plan axial $S_{2}$ pénétrative qui porte une linéation d'étirement plongeante vers l'est à sudest (Fig. 3a). Cette schistosité est accompagnée d'indicateurs cinématiques dont les plus communs correspondent aux structures S-C (Berthé et al., 1979; Lister et Snoke, 1984), développées essentiellement dans les lithologies semipélitiques (Fig. 5d), et aux structures sigmoïdales englobées par les porphyroblastes de grenat (Fig. 5e) et de biotite développés en 
contexte rotationnel. Le sens de cisaillement défini suggère un déplacement de la formation des Skhour de l'est à sudest vers l'ouest à nordouest (Fig. 5d, e).

\section{Structures associées à la tectonique extensive}

La tectonique extensive a donné naissance à une schistosité $S_{2}$ pénétrative, orientée parallèlement aux plans de schistosité $S_{2}$ compressifs de la formation des Skhour. Dans les roches fortement cisaillées, cette schistosité évolue vers une foliation mylonitique qui est bien individualisée dans les microconglomérats et les métapélites. Les linéations d'étirement portées par cette foliation sont orientées NE-SW et plongent faiblement vers le nordest (Fig. 3a). Ces fabriques linéaires sont bien développées dans les métaconglomérats où elles sont materialisées par le grand axe des galets (Fig. 5a).

Les principaux indicateurs cinématiques utilisés pour déduire le sens du mouvement extensif sont les suivants:

i) les structures oeillées ('porphyroclast systems'; Passchier et Simpson, 1986) où les porphyroclastes de quartz des microconglomérats déterminent un système de type s;

ii) les structures planaires composites ('composite planar fabrics'; Simpson et Schmid, 1983) ou fabriques S-C (Berthé et al., 1979; Lister et Snoke, 1984);

iii) les figures de croissance syn-cinématique englobées par les porphyroblastes rotationnels (Fig. $5 f)$.

Le sens de cisaillement défini indique un déplacement du bloc supérieur (formation des Skour) du sudouest vers le nordest. En plus de ces indicateurs cinématiques, le jeu normal de la ZCKM est attesté par des critères purement métamorphiques: l'amincissement conditionné par cette zone de cisaillement a provoqué une forte condensation des zones métamorphiques; celle-ci s'est traduite par:

i) la coïncidence de la staurotide avec l'isograde du disthène;

ii) les distances extrêmement faibles, de l'ordre de 250 à $300 \mathrm{~m}$, marquant le passage de la zone de la biotite à la zone du disthène (Fig. 3 ).

\section{ZONE DE CISAILLEMENT DE OULED ZEDNESS (ZCOZ)}

La nature de cette zone est complexe. Sa limite orientale est un chevauchement (Jenny, 1974; Lagarde et Michard, 1986; Aghzer, 1994), alors que l'occidentale est une faille normale ductile (Aghzer, 1994). Au cours des stades compressifs pré- à syn- $D_{2}$, le cisaillement oriental a assuré le chevauchement de la formation dévonienne à viséenne de Ouled Zedness par la formation cambro-ordovicienne des Skhour (Fig. 3). Le cisaillement occidental, quant à lui, a conditionné la mise en place finale des Rehamna orientaux sur les Rehamna centraux durant les stades extensifs tardi $\mathrm{D}_{2}$ (Fig. 3). Le caractère tardif du régime tectonique extensif est indiqué par le tracé cartographique du chevauchement d'Ouled Zedness qui est tronqué vers le sud, selon un angle faible, par la faille normale d'El Broumi (Fig. 3a). Cet ordre chronologique relatif des deux régimes tectoniques, suggérant un amincissement d'une croûte déjà épaissie par une tectonique en compression, est conforme au modele tectonothermal admis pour les ceintures orogéniques affectées par une tectonique extensive (Houseman et England, 1986).

\section{Structures associées à la tectonique compressive}

Les roches situées au niveau et à proximité du chevauchement de la ZCOZ sont affectées par une schistosité $S_{2}$ fortement pénétrative, qui évolue vers une foliation mylonitique dans des couloirs de déformation intense où elle porte une linéation d'étirement plongeante vers l'est à sudest (Fig. 3a). La schistosité $S_{2}$ est plan axial de plis $P_{2}$ de taille centimétrique, préservés localement squs forme de veines quartzeuses correspondant à des charnières fortement aplaties. Ces plis sont orientés N-S à NESW, présentent un faible plongement axial vers le nord à nordest et sont déversés vers l'ouest à nordouest. Le sens de cisaillement, indiqué par les bandes S-C (Fig. 6a) et les structures sigmoïdales des plans de schistosité interne des porphyroblastes rotationnels (Fig. 6b), suggère une translation du bloc supérieur (Rehamna orientaux) de l'est à sudest vers l'ouest à nordouest.

\section{Structures associées à la tectonique extensive}

Elles affectent les lithologies dévoniennes à viséennes qui se situent au niveau et à proximité de la faille normale de la ZCOZ. Ces structures définissent également une bande étroite de déformation intense, au contact de la formation des Skhour avec la ZCOZ. Elles correspondent à une schistosité $S_{2}$ pénétrative, qui passe en continuité cartographique avec les plans $S_{2}$ compressifs. Les linéations d'étirement portées par la foliation mylonitique plongent vers le nordest (Fig. 3a).

Quoique la tectonique extensive soit accompagnée de processus de recristallisation intense, des plis $\mathrm{P}_{2}$ associés aux stades compressifs antérieurs sont localement préservés. Isoclinaux et d'orientation axiale N-S à NE-SW, ces plis sont déversés vers l'ouest à nordouest et présentent un faible plongement axial vers le nord à nordest.

Les bandes $\mathrm{S}-\mathrm{C}$ et les figures de croissance syncinématique englobées par des porphyroblastes rotationnels (Fig. 6c) indiquent un déplacement du bloc supérieur (formation de Jorf El Ahmar) du sudouest 

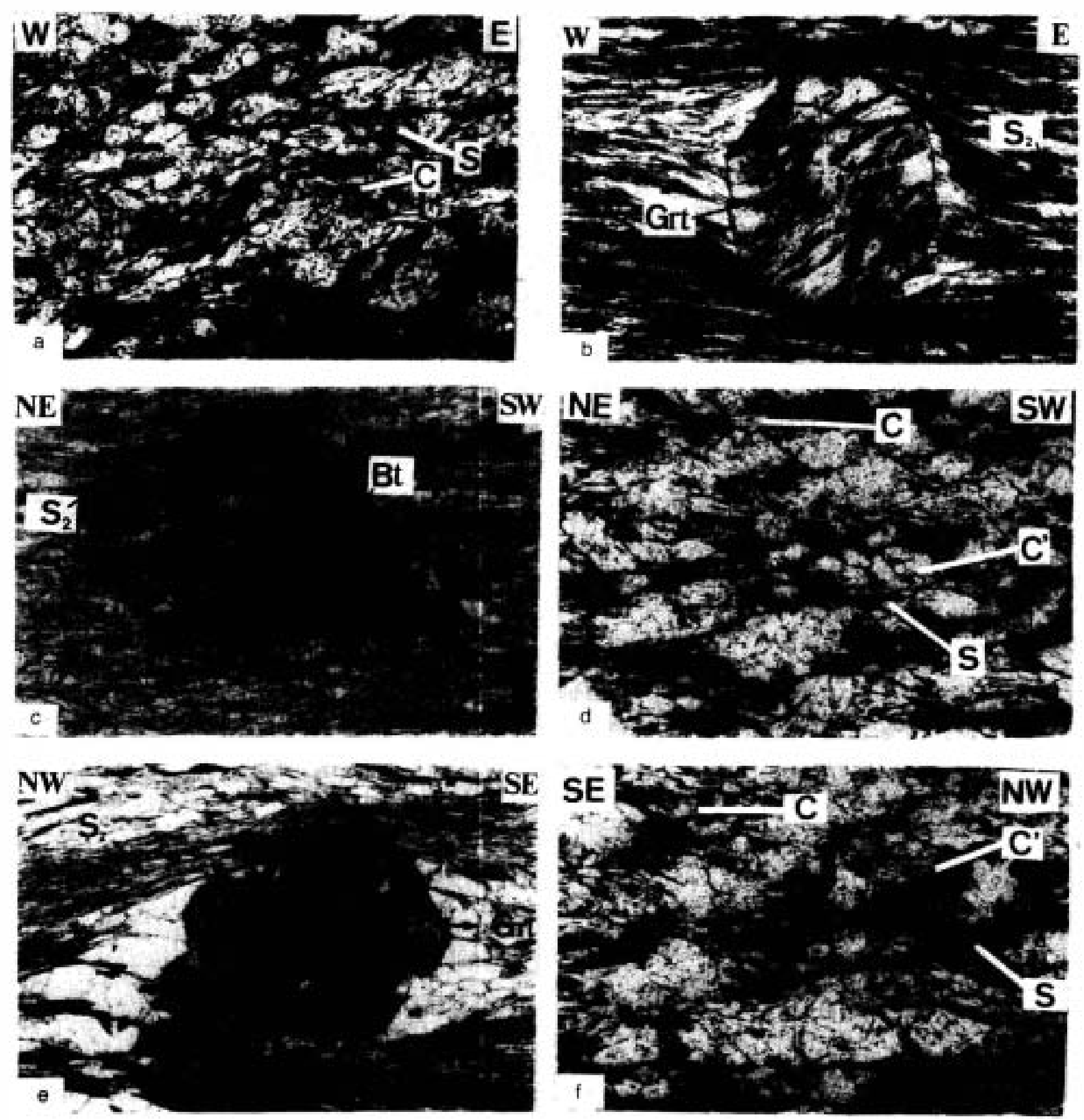

Figure 6. (a) Structures S-C développées dans les métapélites gréseuses localisées au niveau de la limite tectonique orientale de la ZCOZ. Ces structures, engendrées par les stades compressifs de la deuxième phase tectonique, indiquent un déplacement du bloc supérieur (Rehamna orientaux) de l'est vers l'ouest. (b) Grenat syn-S2 compressive, développé dans les métapélites de la limite tectonique orientale de la ZCOZ. Sa schistosité interne adopte une forme sigmoïdale suggérant un déplacement des Rehamna orientaux de l'est vers l'ouest. (c) Biotite porphyro-blastique syn- $S_{2}$ des métapélites de la limite tectonique occidentale de la ZCOZ. Sa croissance en contexte rotationnel indique un transport tectonique du bloc supérieur (formation de Jorf El Ahmar) du sudouest vers le nordest. (d) Structures ECC surimposées aux structures $S-C$ au cours des stades tardifs de la tectonique extensive. Elles affectent les métapélites gréseuses situées au niveau de la limite tectonique occidentale de la ZCOZ. Le sens de cisaillement qu'elles matérialisent indique un déplacement du bloc supérieur du sudouest vers le nordest. (e) Grenat des métapélites de la ZCEB, incluant des figures de rotation syn-cinématique qui suggèrent un déplacement du bloc supérieur (formations d'El Broumi-Ouled Hassine et de Lalla Tittaf) du nordouest vers le sudest. (f) Structures ECC développées dans la ZCEB au cours des stades ultimes de la tectonique extensive. Elles indiquent un sens de cisaillement similaire à celui des structures $S-C$ auquelles elles se superposent, ce qui révèle le jeu en faille normale de la ZCEB et suggère un déplacement du bloc supérieur (formations d'El Broumi-Ouled Hassine et de Lalla Tittaf) du nordouest vers le sudest. Grt-grenat; Btbiotite. La barre noire mesure $150 \mathrm{~mm}$. 
vers le nordest. Des surfaces C' (Berthé et al., 1979; Platt, 1979; Platt et Vissers, 1980; White et al., 1980) ou ECC (Platt et Vissers, 1980; Platt, 1984), indiquant le même sens de cisaillement que celui des fabriques $\mathrm{S}$ $\mathrm{C}$, se superposent tardivement à ces demières tout en formant un angle de $11^{\circ}$ avec les plans $C$ (Fig. 6d).

Le jeu en faille normale de la limite tectonique occidentale de la ZCOZ est également démontré par une étude géobarométrique réalisée dans la formation des Skhour et dans les Rehamna orientaux (Aghzer, 1994). Dans ces deux domaines, les pressions correspondant au pic thermique régional atteint au cours des chevauchements syn- $D_{2}$, montrent des différences de l'ordre de 1,5 kbars. Ces différences de pression suggèrent un rejet vertical de la faille normale de Ouled Zedness de $5 \mathrm{~km}$. Il s'agit en fait d'un rejet minimal établi au cours du refroidissement décompressif conditionné par les stades précoces de la tectonique extensive (Aghzer, 1994). Au niveau de cette limite tectonique, on note en plus que l'isograde de la staurotide coïncide avec la présence du disthène (Fig. 3a). Cette coïncidence, témoignant d'un amincissement crustal intense, confirme davantage le caractère soustractif de ce contact tectonique.

\section{ZONE DE CISAILLEMENT D’EL BROUMI (ZCEB)}

Cette zone de cisaillement superpose la formation dévonienne d'El Broumi-Ouled Hassine sur la formation cambro-ordovicienne de Jorf El Ahmar (Fig. 3a). Les métapélites et semipélites qui y sont localisées sont affectées par une schistosité $S_{2}$ pénétrative. Entre les plans $S_{2}$ on observe localement la schistosité $S_{1}$ plissée au cours de la tectonique compressive $D_{2}$. Les plis $\mathrm{P}_{2}$ sont orientés $\mathrm{N}-\mathrm{S}$ à NE-SW, présentent un faible plongement axial vers le nord à nordest et sont déversés vers l'ouest à nordouest.

Les plans de schistosité $S_{2}$ de la ZCEB passent en continuité cartographique avec ceux des métasédiments environnants des formations de Jorf El Ahmar et d'El Broumi-Ouled Hassine. Au passage à cette zone de cisaillement, la schistosité $S_{2}$ évolue vers une foliation mylonitique. Celle-ci est progressivement acquise par réduction de la taille des grains qui s'opère parallèlement à un enrichissement en matériaux phyllosilicatés. Les linéations d'étirement portées par la foliation $S_{2}$ plongent vers le sudest (Fig. 3a). Les critères $\mathrm{du}$ sens de cisaillement (Fig. 6e), suggérant un déplacement du bloc supérieur (formations d'El Broumi-Ouled Hassine et de Lalla Tittaf) du nordouest vers le sudest, revèlent le jeu en faille normale de la ZCEB. Le caractère soustractif de cette faille est également indiqué par des structures ECC (Platt et Vissers, 1979; Platt, 1984) (Fig. 6f), correspondant aux manifestations ultimes de la tectonique extensive; elles se superposent aux fabriques $\mathrm{S}-\mathrm{C}$ et forment un angle de $15^{\circ}$ avec les plans $\mathrm{C}$. Il est à remarquer que ce sens de ci- saillement est distinct de celui déterminé dans la ZCKM et la ZCOZ. On assiste donc à une variation cinématique des mouvements extensifs dont les données disponibles actuellement ne permettent pas de l'aborder en termes de chronologie relative. L'existence de deux directions d'extension, notamment dans le cas des dômes gneissiques extensifs où l'une des directions est associée à la cinématique du détachement et l'autre au dôme proprement dit (Brun et van den Driessche, 1994), est un phénomène bien connu en Sardaigne (Musumeci, 1992), dans le Massif Central français (van den Driessche et Brun, 1992; Brun et van den Driessche, 1994) et dans le Massif Ibérien (Escuder Viruete et al., 1994; Arenas et al., 1995).

\section{CONCLUSIONS}

Le massif des Rehamna est affecté par une importante tectonique extensive qui a assuré le retour à l'équilibre de la croûte antérieurement épaissie par un régime tectonique compressif. Cette extension crustale s'est manifestée par l'intermédiaire de zones de cisaillement ductile (ZCKM, ZCEB et limite occidentale de la $\mathrm{ZCOZ}$ ) correspondant à des niveaux de détachement post-pic thermique régional. Ces failles de détachement ont engendré des fabriques planaires pénétratives, globalement parallèles en orientation à celles associées à la tectonique en compression. Ces fabriques extensives sont accompagnées de marqueurs cinématiques indiquant une variation du sens des mouvements extensifs avec la profondeur: le sens de déplacement dirigé du sudouest vers le nordest dans les parties profondes de la chaine (formations des Skhour et de Jorf El Ahmar), s'opère par contre $\mathrm{du}$ nordouest vers le sudest au sommet de l'édifice orogénique (formations $d^{\prime} E l$ Broumi-Ouled Hassine et de Lalla Tittaf). Comme conséquence de l'amincissement crustal provoqué par la tectonique extensive, les isogrades ont subi une forte condensation qui est spectaculaire au niveau des zones de détachement, et plus particulièrement dans la ZCKM où le passage de la zone de la biotite à la zone du disthène se réalise sur une distance de 250 à $300 \mathrm{~m}$.

Les processus extensifs mis en évidence dans le massif des Rehamna ont été signalés dans d'autres chaînes, comme dans le Massif Ibérien (Escuder Viruete et al., 1994; Arenas et al., 1995), les Cordillères nord-américaines (Davis, 1980; Malavieille, 1987; Sonder et al., 1987), les Hellénides (Lister et al., 1984; Faure et Bonneau, 1988; Buick et Holland, 1989), les Alpes (Selverstone, 1988) et le Massif Central français (Mattauer et al., 1988; van den Driessche et Brun, 1989; Malavieille et al., 1990). Dans ces chaînes, le rôle de la tectonique extensive, en tant que processus de dénudation des ceintures orogéniques, s'est avéré, comme dans le cas du massif des Rehamna, déterminant dans la ré-équilibration des croûtes épaissies. 


\section{Remerciements}

Nous remercions vivement J. R. Martínez Catalán pour ses discussions enrichissantes pendant la réalisation de ce travail, et $\mathrm{M}$. Corsini et $\mathrm{K}$. Theunissen pour la révision d'une première version du manuscrit. Le premier auteur a beneficié d'une bourse d'études de l'Instituto Español de Cooperación con El Mundo Arabe'.

\section{REFERENCES}

Aghzer, A. M. 1994. Evolution tectonothermale du massif hercynien des Rehamna (Zone mésétienne centrale, Maroc). Thèse 358p. Université Complutense, Madrid.

Arenas, R., Rubio Pascual, F. J., Díaz García, F. et Martínez Catalán, J. R. 1995. High-pressure microinclusions and development of an inverted metamorphic gradient in the Santiago Schists (Ordenes Complex, NW Iberian Massif, Spain): evidence of subduction and syn-collisional decompression. Journal Metamorphic Geology 13, 141-164.

Berthé, D., Choukroune, P. et Jegouzou, P. 1979. Orthogneiss, mylonite and non-coaxial deformation of granites: the example of the south Armorican Shear Zone. Journal Structural Geology 1, 31-42.

Brun, J. P. et van den Driessche, J. 1994. Extensional gneiss domes and detachment fault systems: structure and kinematics. Bulletin Société Géologique France 6, 519-530.

Buick, I. S. et Holland, T. J. B. 1989. The P-T-t paths associated with crustal extension, Naxos, Cyclades, Greece. In: Evolution of Metamorphic Belts (Edité par Daly, J. S., Cliff, R. A. et Yardley, B. W. D.). Geological Society Special Publication 43, 365-369.

Coney, R. P. J. 1984. The regional tectonic setting and possible causes of Cenozoic extension in the North American Cordillera. In: Continental Extensional Tectonics (Edité par Coward, M. P., Dewey, J. E. et Hancock, P. C.). Geological Society London Special Publication 28, 177-186.

Cornée, J. J., Muller, J. et Sougy, J. 1982. Styles et âges de mise en place des nappes hercyniennes dans le massif des Rehamna orientales (Maroc). Comptes Rendus Académie Sciences Paris 294, 1107-1110.

Corsini, M. 1988. Lithostratigraphie et tectonique des terrains paléozoïques dans les Rehamna et la Méséta côtière. (Meseta marocaine hercynienne). Thèse 150p. Université Aix-Marseille,

Corsini, M., Cornée, J. J., Muller, J. et Vauchez, A. 1988. Cisaillement synmétamorphe et déplacement tangentiel vers le SW dans les Rehamna (Maroc hercynien). Comptes Rendus Académie Sciences Paris 306, 1389-1394.

Davis, G. H. 1980. Structural characteristics of metamorphic complexes. In: Cordilleran Metamorphic Core
Complexes (Edité par Crittenden, M. D. Jr, Coney, P. J. et Davis, G. H.). Journal Geological Society America 153, 79-129.

Destombes, J., Guezou, J. C., Hoeppfner, C., Jenny, P., Piqué, A. et Michard, A. 1982. Le primaire du massif des Rehamna s.str.; problèmes de stratigraphie des séries métamorphiques. Notes Mémoires Service Géologique Maroc 303, 35-70.

Diot, H. 1989. Mise en place des granitoïdes hercyniens de la Mesta marocaine. Thèse 163p. Université Toulouse,

Escuder Viruete, J. E., Arenas, R. et Martínez Catalán, J. R. 1994. Tectonothermal evolution associated with Variscan crustal extension in the Tormes Gneiss Dome (NW Salamanca, Iberian Massif, Spain). Tectonophysics 238, 1-22.

Faure, M. et Bonneau, M. 1988. Données nouvelles sur l'extension néogène de l'Egée: la déformation du granite de Mykonos (Cyclades, Grèce). Comptes Rendus Académie Sciences Paris 307, 1553-1559.

Hodges, K. V., Walker, J. D. et Wernick, B. P. 1987. Footwall structural evolution of Tucki Mountain detachment system, Death Valley region, Southeastern California. In: Continental Extensional Tectonics (Edité par Coward, M. P., Dewey, J. E. et Hancock, P. L.). Geological Society London, Special Publication 28, 393-408.

Hoeppfner, C. 1974. Contribution à la géologie structurale des Rehamna (Méséta marocaine méridionale); le matériel paléozoïque et son évolution hercynienne dans l'Est du Massif. Thèse $3^{\text {ème }}$ cycle 92p. Université Louis-Pasteur, Strasbourg.

Hoeppfner, C., Jenny, P. et Piqué, A. 1975. La tectogénèse hercynienne dans le massif des Rehamna (Maroc). Ecaillages et coulissements synmétamorphiques en bordure du môle côtier mésétien. Sciences Géologiques Bulletin Strasbourg 3, 179-203.

Houseman, G. et England, P. 1986. A dynamic model of lithosphere extension within the Higher Himalayas (Ladakh, India). Geology 15, 409-413.

Jenny, P. 1974. Contribution à la géologie structutrale des Rehamna (Méséta marocaine méridionale); le matériel paléozoïque et son évolution hercynienne dans le centre du massif. Thèse $3^{\text {ine }}$ cycle $120 \mathrm{p}$. Université Louis-Pasteur, Strasbourg.

Lagarde, J. L. et Michard, A. 1986. Stretching normal to the regional thrust displacement in a thrustwrench shear zone. Rehamna Massif, Morocco. Journal Structural Geology 8, 483-492.

Lister, J. C. et Snoke, A. W. 1984. S-C-mylonites. Journal Structural Geology 6, 617-638.

Lister, G., Banga, G. et Feenstra, A. 1984. Metamorphic core complexes of Cordilleran type in the cyclades, Aegean Sea, Greece. Geology 12, 221-225.

Malavieille, J. 1987. Kinematics of compressional and extensional ductile shearing deformation in a metamorphic core complex of the Northeastern ba- 
sin \& range. Journal Structural Geology 9, 541-554.

Malavieille, J., Guihlot, P., Costa, S., Lardeaux, J. M. et Garden, V. 1990. Collapse of the thickened Variscan crust in the French Massif Central: Mount Pilat extensional shear zone and St Etienne late Carboniferous basin. Tectonophysics 177, 139-149.

Mattauer, M., Brunel, M. et Matte, P. 1988. Failles normales ductiles et grands chevauchements. Une nouvelle analogie entre l'Himalaya et la chaîne hercynienne du Massif Central français. Comptes Rendus Académie Sciences Paris 306, 671-676.

Michard, A. 1982. Le massif paléozoïque des Rehamna (Maroc). Stratigraphie, tectonique et pétrogénèse d'un segment de la chaîne varisque. Notes Mémoires Service Géologique Maroc 303, 180p.

Musumeci, G. 1992. Ductile wrench tectonics and exhumation of hercynian metamorphic basement in Sardinia: Monte Grighini Complexe. Geodinamica Acta 5, 119-133.

Passchier, C. W. et Simpson, C. 1986. Porphyroclast systems as kinematic indicators. Journal Structural Geology 8, 831-843.

Piqué, A. 1972. Contribution à la géologie structurale des Rehamna (Méséta marocaine méridionale). Le matériel paléozoïque et son évolution hercynienne dans l'Ouest du massif. Thèse $3^{\text {ème }}$ cycle 101p. Université Louis-Pasteur, Strasbourg.

Piqué, A. et Michard, A. 1989. Moroccan hercynides: a synopsis. The Paleozoic sedimentary and tectonic evolution at the northern margin of West Africa. American Journal Science 289, 286-330.

Piqué, A., Hoeppfner, C., Jenny, J., Guezou, J. C. et Michard, A. 1982. Tectonique du massif hercynien des Rehamna (Maroc). Evolution de la déformation dans les zones métamorphiques hercyniennes.
Notes Mémoires Service Géologique Maroc 303, 86-129.

Platt, J. P. 1979. Extensional crenulation cleavage. Journal Structural Geology 1, 95.

Platt, J. P. 1984. Secondary cleavages in ductile shear zones. Journal Structural Geology 6, 439-442.

Platt, J. P. et Vissers, R. L. M. 1980. Extensional structures in anisotropic rocks. Journal Structural Geology 2, 397-415.

Sandiford, M. 1989. Horizontal structures in granulite terrains: a record of mountain building or mountain collapse? Geology 17, 449-453.

Selverstone, J. 1988. Evidence for East-West crustal extension in the eastern Alps. Implications for the unroofing history of the Tauern Window. Tectonics 7, 87-105.

Simpson, C. et Schmid, S. M. 1983. An evaluation of criteria to deduce the sense of movement in sheared rocks. Geological Society America Bulletin 94, 1281-1288.

Sonder, L. J., England, P. C., Wernicke, B. P. et Christiansen, R. L. 1987. A physical model for Cenozoic extension of Western North America. In: Continental Extensional Tectonics (Edité par Coward, M. P., Dewey, J. E. et Hancock, P. L.). Geological Society London, Special Publication 28, 177-186.

Van den Driessche, J. et Brun, J. P. 1989. Un modèle cinématique de l'extension paléozoïque supérieure dans le Sud du Massif Central. Comptes Rendus Académie Sciences Paris 309, 1607-1613.

Van den Driessche, J. et Brun, J. P. 1992. Tectonic evolution of the Montagne Noire (French Massif Central): a model of extensional gneiss dome. Geodinamica Acta 5, 85-92.

White, S. H., Burrows, S. E., Carreras, J., Shaw, N. D. et Humphrey, F. J. 1980. On mylonites in ductile shear zones. Journal Structural Geology 2, 175-187. 